\title{
MICORRIZAÇÃo E ÉPOCAS DE DESSECAÇÃo DE Brachiaria brizantha NO DESENVOLVIMENTo DA SOJA ${ }^{1}$
}

\author{
Mycorrhizal Inoculation and Desiccation Timings of Brachiaria brizantha on Soybean \\ Development
}

\begin{abstract}
SILVA, A.C. ${ }^{2}$, SANTOS, J.B. ${ }^{3}$, KASUYA, M.C.M. ${ }^{4}$, SILVA, A.A. ${ }^{5}$ e MANABE, A. ${ }^{6}$
RESUMO - Por meio deste trabalho objetivou-se avaliar diferentes intervalos entre a dessecação química ou roçada de Brachiaria brizantha, inoculada ou não com fungos micorrízicos arbusculares, e a semeadura da soja. B. brizantha foi semeada em vasos na presença ou ausência de inóculo misto dos fungos Glomus etunicatum, Glomus fasciculatume Scutellospora heterogama. A gramínea foi semeada escalonadamente, de forma que apresentasse o mesmo estádio de desenvolvimento por ocasião da dessecação química ou roçada, aos $7,14,21$ ou 28 dias antes da semeadura (DAS) da soja. A soja, semeada em única época, foi inoculada com estirpes de Bradyrhizobium (SEMIA 5019, SEMIA 5079, SEMIA 5080 e SEMIA 587). A avaliação da soja foi feita no estádio de pleno florescimento. Não se observou efeito do herbicida sobre a soja quando a dessecação foi realizada aos 14, 21 ou 28 DAS; entretanto, em todas as épocas, a cultura desenvolveu-se melhor quando $B$. brizantha foi inoculada com os fungos micorrízicos. A dessecação, realizada aos 7 DAS, proporcionou maiores área foliar e número de trifólios e de nódulos, assim como de biomassa seca de folhas da soja, em relação ao tratamento roçado. Todavia, nessa época, verificou-se decréscimo na colonização micorrízica e no volume das raízes da soja.
\end{abstract}

Palavras-chave: Bradyrhizobium, herbicida, micorrizas arbusculares, nodulação.

\begin{abstract}
This study aimed to evaluate different intervals between chemical desiccation or cut Brachiaria brizantha, inoculated or not with arbuscular mycorrhizal fungi, and soybean sowing. B. brizantha was sown in pots, in the presence or absence of Glomus etunicatum, Glomus fasciculatum and Scutellospora heterogama mixture. The grass was sown in different periods so as to present the same developmental stage at the time of chemical desiccation or cut, at 7, 14, 21 or 28 days before soybean sowing (DBS). Soybean sown in a single time was inoculated to a mixture of Bradyrhizobium strains (SEMIA 5019, SEMIA 5079, SEMIA 5080, and SEMIA 587). The soybean plant was evaluated at the flowering stage. No herbicide effect was verified on the soybean when desiccation was performed at 14, 21 or 28 DBS; however, at all times, soybean developed better when B. brizantha was mycorrhizal fungi inoculated. Desiccation at 7 DBS increased of leaf area, trifoliate and nodule number, and soybean leaf dry biomass, compared to the cut treatment. However, at this same time, mycorrhizal colonization and soybean root volume were found to decrease.
\end{abstract}

Keywords: Bradyrhizobium, herbicide, arbuscular mycorrhiza, nodulation.

Recebido para publicação em 1.2.2005 e na forma revisada em 5.5.2006.

2 Pesquisadora da Agência Paulista de Tecnologia dos Agronegócios - APTA, Pólo Regional da Alta Sorocabana, Rod. Raposo Tavares, km 561, Caixa Postal 298, 19015-970 Presidente Prudente-SP, <andreiacs@ aptaregional.sp.gov.br>. ${ }^{3}$ Pós-Doutor do Dep. de Fitotecnia da Universidade Federal de Viçosa - UFV; ${ }^{4}$ Professora do Dep. de Microbiologia da UFV; ${ }^{5}$ Professor do Dep. de Fitotecnia da UFV; ${ }^{6}$ M.S. em Microbiologia do Solo pela UFV.

Planta Daninha, Viçosa-MG, v. 24, n. 2, p. 271-277, 2006 


\section{INTRODUÇÃO}

O cultivo da soja tem sido uma das principais alternativas para a recuperação e renovação de pastagens degradadas (Kichel et al., 2000), sendo Brachiaria brizantha cv. Marandu, lançada pela Embrapa na década de 1980, a espécie forrageira que contempla mais de 50\% do mercado nacional (Zimmer \& Euclides Filho, 1997). A braquiária é dessecada com herbicidas de manejo, como o glyphosate, formando cobertura morta para o plantio direto da soja.

É notório que o aproveitamento dos fungos micorrízicos arbusculares para o aumento de produtividade das pastagens tropicais é uma alternativa bastante promissora. As micorrizas arbusculares são de ocorrência generalizada, em especial nos trópicos, infectando a maioria das plantas (Souza et al., 1999), o que é extremamente benéfico. A interferência negativa do glyphosate sobre microrganismos e também sobre a associação com as plantas vem sendo investigada (Kaps \& Kuhns, 1987; Edwards, 1989; Chakravarty \& Chatarpaul, 1990; Moorman \& Dowler, 1991; Santos et al., 2003, 2004, 2005).

O glyphosate é um composto de baixa volatilidade e insignificante fotólise. A degradação microbiana constitui-se no principal processo de dissipação desse produto no solo (Amarante Junior \& Santos, 2002). Esse herbicida, ao entrar em contato com o solo, desde que disponivel em solução aquosa e não adsorvido em colóides, é rapidamente biodegradado por fungos, bactérias e actinomicetos, com subseqüente diminuição de sua persistência no meio ambiente (Ahrens, 1994). No entanto, relatos de toxicidade desse herbicida a microrganismos têm sido verificados na literatura (Santos et al., 2003, 2004, 2005).

Quando adsorvido ao solo, o glyphosate não causa intoxicação a espécies vegetais cultivadas após sua aplicação. Contudo, na presença de fungos micorrízicos, que realizam a interação fungo-planta, hifas de um mesmo fungo podem promover a união de mais de uma espécie vegetal (Kaps \& Kuhns, 1987). Nessa situação, o glyphosate, quando aplicado em uma espécie, poderia afetar o fungo micorrízico e a outra espécie vegetal envolvida. Em experimentos realizados para verificar tal hipótese, Kaps \& Kuhns (1987) aplicaram glyphosate com carbono marcado $\left(\mathrm{C}^{14}\right) \mathrm{em}$ solução nutritiva onde cresciam plântulas de Pinus spp., transferindo-as, posteriormente, para crescimento junto a espécies de plantas daninhas. Os autores observaram, pela análise radiográfica, moléculas de glyphosate nas espécies daninhas, evidenciando a comunicação radicular entre o Pinus spp. e as espécies por meio de micorrizas.

Outro aspecto importante, em se tratando da simbiose micorrízica na cultura da soja, é a interação fungo micorrízico-rizóbio. São relatados efeitos positivos dessa interação (Pacovsky et al., 1986), resultado de melhor nutrição, como, por exemplo, o fornecimento de fósforo às raízes (Bethlenfalvay \& Yoder, 1981), e/ou garantindo o suprimento desse nutriente aos bacterióides (Bergersen, 1971).

Objetivou-se com este trabalho avaliar diferentes intervalos entre a dessecação química ou roçada de $B$. brizantha, inoculada ou não com fungos micorrízicos arbusculares, e a semeadura da soja sobre o desenvolvimento inicial da cultura.

\section{MATERIAL E MÉTODOS}

O experimento foi realizado em casa de vegetação, com ambiente controlado, pertencente ao Laboratório de Associações Micorrízicas do Departamento de Microbiologia da UFV, no período de novembro de 2003 a fevereiro de 2004.

O delineamento experimental adotado foi o de blocos casualizados, com 16 tratamentos e quatro repetições, em arranjo fatorial $2 \times 2 \times 4$, consistindo na inoculação ou não de $B$. brizantha com os fungos micorrízicos vesículo-arbusculares (MA) Glomus etunicatum, G. fasciculatum e Scutellospora heterogama e dois manejos de $B$. brizantha (dessecação química ou roçada), realizados em quatro épocas $(7,14,21$ ou 28 dias antes do plantio da soja DAP).

B. brizantha foi semeada em vasos contendo $3 \mathrm{dm}^{3}$ de areia, previamente esterilizada, de forma a obter 30 plantas por vaso. A semeadura foi feita a intervalos de sete dias, de forma que a dessecação ou roçada ocorresse aos 7 , 14, 21 ou 28 dias antes da semeadura da soja. 
As plantas foram dessecadas (glyphosate a $1,44 \mathrm{~L} \mathrm{ha}^{-1}$ ) ou roçadas (cortadas rente ao solo) aos 45 dias após a semeadura.

A dessecação química foi feita empregando-se pulverizador costal de precisão, utilizando-se bico TT110.02, à pressão constante de $3,0 \mathrm{kgf} \mathrm{cm}^{-2}$, aplicando-se o equivalente a $100 \mathrm{~L} \mathrm{ha}^{-1}$ de calda.

A semeadura da soja foi realizada sete dias após a última dessecação ou roçada. As sementes de soja, cultivar UFV-16, foram desinfestadas superficialmente com hipoclorito de sódio a $2 \%$ por cinco minutos, sendo posteriormente lavadas e mergulhadas em meio contendo mistura de quatro estirpes de Bradyrhizobium (SEMIA 5019, SEMIA 5079, SEMIA 5080 e SEMIA 587), em fase ativa de crescimento. A unidade experimental foi representada por um vaso contendo duas plantas de soja.

Durante todo o experimento a suplementação mineral foi realizada pela adição de solução de Clark (Clark, 1975), que apresenta as seguintes concentrações de macronutrientes: 5,$7 ; 1,0 ; 0,1 ; 2,4 ; 1,2 ; 0,6$; e $0,7 \mathrm{mmol} \mathrm{L}^{-1}$ de $\mathrm{N}-\mathrm{NO}_{3}^{-}, \mathrm{N}-\mathrm{NH}_{4}^{+}, \mathrm{P}, \mathrm{K}, \mathrm{Ca}^{2+}, \mathrm{Mg}^{2+}$ e S, respectivamente. As concentrações de micronutrientes foram de 19,0, 0,5; 40,0; 7,0; 0,09; e 2,0 $\mu \mathrm{mol} \mathrm{L}^{-1}$ de $\mathrm{B}, \mathrm{Cu}, \mathrm{Fe}, \mathrm{Mn}$, Mo e $\mathrm{Zn}$, respectivamente. O fornecimento da solução nutritiva foi realizado semanalmente; na fase inicial de desenvolvimento, tanto de $B$. brizantha quanto da soja (aproximadamente até os 20 dias após a semeadura - DAS), o fornecimento se deu com $50 \%$ de concentração dos nutrientes - "meia-força". O pH e a condutividade elétrica foram monitorados de maneira a evitar problemas na absorção dos nutrientes pelas plantas.

As plantas de soja foram colhidas no estádio fenológico de pleno florescimento (R2), segundo escala de Fehr et al. (1971), sendo avaliados: biomassa seca de folhas e raízes, altura de plantas, número e biomassa fresca e seca de nódulos, área foliar, volume de raízes, porcentagem de colonização micorrízica e número de trifólios. Para determinação da porcentagem de colonização micorrízica, as raízes foram clarificadas com $\mathrm{KOH}$ a $10 \%$, coradas em lactoglicerol contendo azul-detripano (Schenck, 1982) e dispostas sobre uma placa de Petri com o fundo quadriculado, onde cada segmento de raiz contido sobre uma linha do quadrículo foi somado como micorrizado ou não, respectivamente, pela presença ou ausência de estruturas fúngicas.

Os dados foram submetidos à análise de variância, sendo as médias dos tratamentos comparadas dentro de cada época. Para as diferentes épocas de dessecação, foram ajustadas curvas de regressão.

\section{RESULTADOS E DISCUSSÃO}

Em todas as características avaliadas verificou-se efeito positivo da inoculação com fungos micorrízicos arbusculares. Foi observado melhor desenvolvimento das plantas de soja onde previamente se cultivou B. brizantha inoculada com Glomus etunicatum, G. fasciculatum e Scutellospora heterogama (Tabela 1 e Figuras 1 e 2).

No manejo realizado aos 7 DAS, a utilização do glyphosate para dessecação química de $B$. brizantha diferiu da roçada para volume de raízes, biomassa seca de folhas, área foliar, número de nódulos e trifólios e porcentagem de colonização micorrízica (Tabela 1). Para as demais características avaliadas, não houve efeito do manejo prévio de $B$. brizantha (dessecada ou roçada) em qualquer época (Figuras 1 e 2). Alguns autores relataram indiferença da micorrização na presença de glyphosate (Chakravarty \& Chatarpaul, 1990), porém trabalhos mostram redução ou inibição no processo de micorrização (Eberbach \& Douglas, 1989) e nodulação da soja (Moore et al., 1983).

Observou-se interação entre épocas de dessecação ou roçada $(7,14,21$ ou 28 DAP) e a micorrização (inoculada ou não) de $B$. brizantha para a produção de biomassa seca das raízes e da parte aérea da soja (Figura 1). Plantas de soja que se desenvolveram em vasos onde houve prévia micorrização apresentaram o mesmo comportamento, independentemente da época de dessecação ou roçada de $B$. brizantha. Entretanto, nos tratamentos em que $B$. brizantha não foi inoculada, houve redução de biomassa seca da raiz e da parte aérea nas plantas de soja (explicada por equação polinomial não-linear $\mathrm{P}>0,01$ ) à medida que se diminuiu o tempo entre a dessecação ou 
Tabela 1 - Volume de raízes, área foliar, número de trifólios e de nódulos, biomassa seca de folhas e porcentagem de colonização micorrízica de plantas de soja, no estádio de pleno florescimento, crescidas em vasos onde previamente se cultivou Brachiaria brizantha inoculada ou não com fungos endomicorrízicos e dessecada ou roçada, aos sete dias antes da semeadura

\begin{tabular}{|c|c|c|c|c|}
\hline \multirow{2}{*}{$\begin{array}{c}\text { Fungo micorrízico em } \\
\text { B. brizantha }\end{array}$} & \multicolumn{2}{|c|}{ Volume de raízes $\left(\mathrm{cm}^{3}\right)$} & \multicolumn{2}{|c|}{ Área foliar $\left(\mathrm{cm}^{2}\right)$} \\
\hline & Dessecada & Roçada & Dessecada & Roçada \\
\hline Inoculada & 17,02 a $\mathrm{B}$ & 22,62 a $\mathrm{A}$ & 364,23 a $\mathrm{A}$ & 323,60 a $\mathrm{B}$ \\
\hline \multirow[t]{3}{*}{ Não-inoculada } & $12,87 \mathrm{~b} \mathrm{~A}$ & $12,50 \mathrm{~b} \mathrm{~A}$ & $104,46 \mathrm{~b} \mathrm{~A}$ & $118,12 \mathrm{~b} \mathrm{~A}$ \\
\hline & \multicolumn{2}{|c|}{ Número de trifólios } & \multicolumn{2}{|c|}{ Biomassa seca de folhas (g) } \\
\hline & Dessecada & Roçada & Dessecada & Roçada \\
\hline Inoculada & 7,67 a $\mathrm{A}$ & 6,50 a B & 1,90 a $\mathrm{A}$ & 1,67 a B \\
\hline \multirow[t]{3}{*}{ Não-inoculada } & $4,04 \mathrm{~b} \mathrm{~A}$ & $3,67 \mathrm{~b} \mathrm{~A}$ & $0,47 \mathrm{~b} \mathrm{~A}$ & $0,57 \mathrm{~b} \mathrm{~A}$ \\
\hline & \multicolumn{2}{|c|}{ Número de nódulos } & \multicolumn{2}{|c|}{ Colonização micorrízica (\%) } \\
\hline & Dessecada & Roçada & Dessecada & Roçada \\
\hline Inoculada & 66,25 a A & 53,00 a B & $31,75 \mathrm{~B}$ & $55,50 \mathrm{~A}$ \\
\hline Não-inoculada & $20,00 \mathrm{~b} \mathrm{~A}$ & $18,87 \mathrm{~b} \mathrm{~A}$ & - & - \\
\hline
\end{tabular}

Médias seguidas por letras iguais, maiúsculas na linha ou minúsculas na coluna, para cada característica, não diferem entre si pelo teste $\mathrm{F}$ a $5 \%$ de probabilidade de erro.

roçada e a semeadura dessa cultura (Figura 1). Esse comportamento pode ser explicado pelo efeito mecânico das raízes e folhas de $B$. brizantha no estabelecimento do sistema radicular da soja e, conseqüentemente, da parte aérea. Quanto maior o intervalo entre a dessecação ou roçada e a semeadura da soja, menor foi o efeito físico da forrageira, devido ao processo de decomposição da palhada. Os tratamentos não-micorrizados podem ter sofrido mais o efeito mecânico dos restos culturais de $B$. brizantha que as plantas micorrizadas, em razão da maior eficiência na ocupação do espaço pelas raízes das plantas micorrizadas, as quais podem ter se estabelecido melhor mesmo com os restos remanescentes de $B$. brizantha.

Para as biomassas fresca e seca de nódulos e a altura das plantas de soja, não houve interação entre épocas de dessecação ou roçada e micorrização (inoculada ou não) de $B$. brizantha. As biomassas fresca e seca de nódulos e a altura das plantas foram constantes ao longo das diferentes épocas de dessecação ou roçada tanto para os tratamentos micorrizados como para os não-micorrizados. A inoculação prévia dos fungos promoveu incremento na biomassa de nódulos e na altura das plantas (Figura 2). Conforme já relatado, a associação de fungos micorrízicos às raízes da soja resulta em maior eficiência no processo de nodulação (Bergensen, 1971; Bethlenfalvay
\& Yoder, 1981; Pacovsky et al., 1986). Trabalhos com soja demonstram que as plantas inoculadas com fungos micorrízicos absorvem maiores quantidade de nutrientes do solo; além disso, a micorrização beneficia a nodulação e o processo de fixação biológica de N (Lopes \& Siqueira, 1981), havendo, conseqüentemente, maior produção de matéria seca da parte aérea e de grãos (Paula et al., 1988).

Na Tabela 1 observa-se que, na última época de dessecação ou roçada de $B$. brizantha (7 DAS), a aplicação de glyphosate resultou em efeito sobre os tratamentos onde se inoculou $B$. brizantha com fungos micorrízicos, convertendo-se em decréscimo do volume de raízes de soja $(22,62$ e 17,02 g para $B$. brizantha roçada e dessecada, respectivamente), evidenciando assim efeito inibitório na micorrização pelo herbicida. Nos tratamentos em que $B$. brizantha foi inoculada com os fungos endomicorrizicos, o glyphosate somente afetou a porcentagem de colonização das raízes de soja quando aplicado aos 7 DAS. Nessa época, foram observados $55,5 \%$ de colonização de raízes de soja onde $B$. brizantha foi roçada e $31,75 \%$ quando foi dessecada com glyphosate. Nas demais épocas não foram observados efeitos da dessecação sobre a colonização das raízes. É provável que a diminuição da colonização micorrízica contribua para a redução no volume das raízes constatada na última época. 

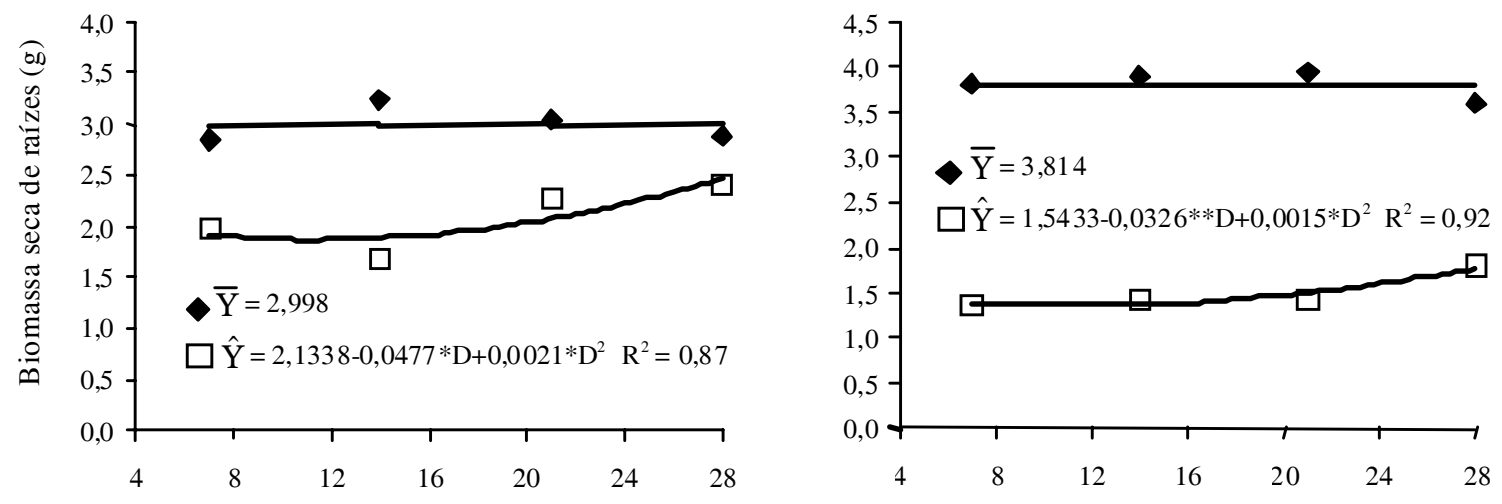

Dias após a dessecação ou roçada

Figura 1 - Biomassa seca de raízes e da parte aérea de plantas de soja crescidas em vasos onde previamente se cultivou $B$. brizantha inoculada $(\diamond)$ ou não-inoculada $(\square)$ com fungos endomicorrízicos e dessecada ou roçada em diferentes épocas antes da semeadura da soja.
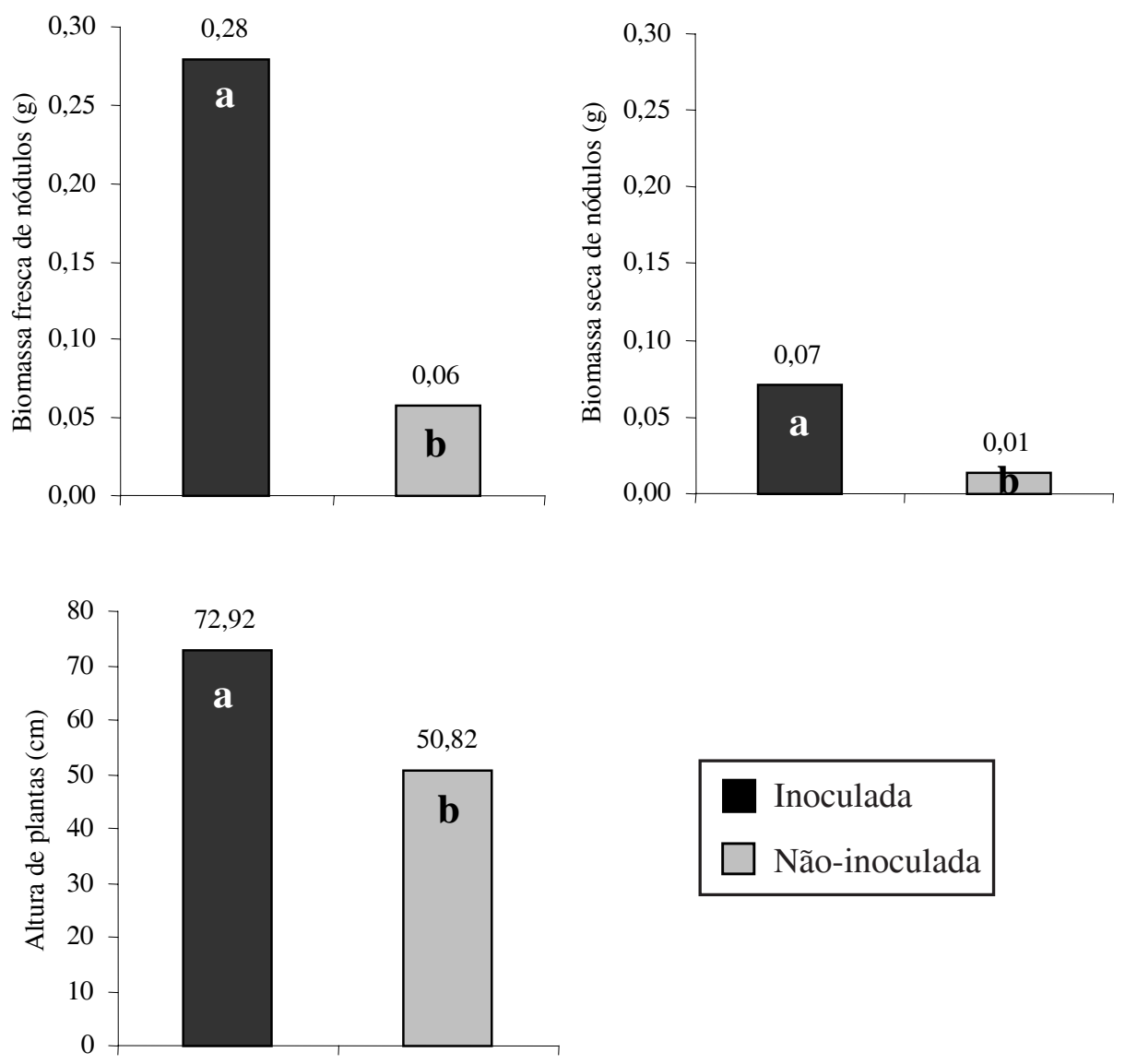

Médias seguidas por letras diferentes para cada característica indicam diferença significativa pelo teste F a 5\% de probabilidade.

Figura 2 - Biomassas fresca e seca de nódulos e altura de plantas de soja, no estádio de pleno florescimento, crescidas em vasos onde previamente se cultivou $B$. brizantha inoculada ou não com fungos endomicorrízicos e dessecada ou roçada em diferentes épocas antes da semeadura da soja. 
A área foliar, o número de trifólios e de nódulos e a biomassa seca das folhas de soja foram incrementados com a aplicação de glyphosate em $11,1 \%, 15,3 \%, 20,0 \%$ e $12,1 \%$, respectivamente, comparado a $B$. brizantha roçada (Tabela 1), nas plantas inoculadas com fungos micorrízicos. Considerando que B. brizantha é uma espécie perene, esse comportamento pode ser explicado pela tendência ao rebrote das plantas roçadas. A presença de raízes ainda vivas representou, além de impedimento físico, maior competição com as plantas de soja, ao passo que as plantas dessecadas apresentavam-se em estágio avançado de decomposição, o que, comparativamente, beneficiou a cultura. É importante ressaltar que o efeito favorável da decomposição mais rápida de $B$. brizantha pelo herbicida foi mais pronunciado devido ao substrato (areia) utilizado. O efeito da dessecação ou roçada foi evidente nas plantas micorrizadas, possivelmente pelo maior desenvolvimento dessas plantas e conseqüente maior demanda pelos fatores de crescimento.

Em condições de campo, a associação de fungos micorrízicos à soja é de extrema importância, visto que, em geral, as leguminosas possuem pêlos radiculares curtos ou escassos, podendo ser mais dependentes de micorrizas para absorverem nutrientes do solo (Baylis, 1975). Essa situação se agrava quando há competição por nutrientes entre a cultura e as plantas daninhas, sendo essencial a maior exploração do solo pelas raízes. Outros trabalhos também demonstraram prejuízos no processo de micorrização em função da aplicação de herbicidas (Berner et al., 1991; Paula Jr. \& Zambolim, 1994).

Tendo em vista os benefícios da micorrização na cultura da soja e a possivel interferência do glyphosate nesse processo, a escolha da época adequada para a dessecação pode diminuir o impacto negativo desse herbicida sobre tal associação. Neste trabalho, o intervalo entre a dessecação química de $B$. brizantha e a semeadura da soja, superior a 14 dias, não interferiu nas características físicas avaliadas.

\section{LITERATURA CITADA}

AHRENS, W. H. Herbicide handbook. 7.ed. Champaign: WSSA, 1994. $352 \mathrm{p}$.

Planta Daninha, Viçosa-MG, v. 24, n. 2, p. 271-277, 2006
AMARANTE JÚNIOR, O. P.; SANTOS, T. C. R. Glifosato: propriedade, toxicidade, usos e legislação. (Revisão). Química Nova, v. 25, n. 4, p. 589-593, 2002.

BAYLIS, G. T. S. The magnolioid mycorrhiza and mycotrophy in root system derived from it. In: SANDERS, F. E.; MOSSE, B.; TINKER, P. B. (Eds.).

Endomycorrhizas. London: Academic Press, 1975. p. 373-389.

BERGERSEN, F. J. Biochemistry of symbiotic nitrogen fixation in legumes. Ann. Rev. Plant Physiol., v. 21, n. 2, p. 121-140, 1971.

BERNER, D. K.; BERGGREN, G. T.; SNOW, J. P. Effects of glyphosate on Calonectia crotalariae and red crown rot of soybean. Plant Dis., v. 75, n. 8, p. 809-813,1991.

BETHLENFALVAY, G. J.; YODER, J. F. The Glycine Glomus - Rhizobium symbioses. I. Phosphorus effects on nitrogen fixation and mycorrhizal infection. Physiol. Plant., v. 52, n. 2, p. 141-145, 1981.

CHAKRAVARTY, P.; CHATARPAUL, L. Non-target effect of herbicides. II. The influence of glyphosate on ectomycorrhizal symbiosis of red pine Pinus resinosa under greenhouse and field conditions. Pestic. Sci., v. 28, p. 243-248, 1990.

CLARK, R. B. Characterization of phosphatase of intact maize roots. J. Agric. Food Chem., v. 23, p. 458-460, 1975.

EBERBACH, P. L.; DOUGLAS, L. A. Herbicide effects on the growth and nodulation potential of Rhizobium trifolii with Trifolium subterraneum. Plant Soil, v. 119, p. 15-23, 1989.

EDWARDS, C. A. Impact of herbicides on soil ecosystems. Crit. Rev. Plant Sci., v. 8, p.221-257, 1989.

FEHR, W. R. et al. Stage of development descriptions for soybeans, Glycine max (L.) Merrill. Crop Sci., v. 11, p. 929-931, 1971.

KAPS, M. A.; KUHNS, L. J. Glyphosate transfer between plants via mycorrhizal fungi. Hortic. Sci., v. 22, n. 5, p. 652, 1987.

KICHEL, A. N.; MIRANDA, C. H. B.; TAMBOSI, S. A. T. Produção de bovinos de corte com a integração agricultura $\mathrm{x}$ pecuária. In: SIMPÓSIO DE FORRAGICULTURA E PASTAGENS: TEMAS EM EVIDÊNCIAS, 1., 2000, Lavras. Anais... Lavras: UFLA, 2000. p. 51-68

LOPES, E. S.; SIQUEIRA, J. O. Vesicular-arbuscular mycorrhizas - their potential in phosphate nutrition in tropical regions. In: RUSSEL, R. S.; IGUE, K.; MEHTA, Y. R. (Eds.). The soil / root system in relation to Brazilian Agriculture. Londrina: IAPAR, 1981. p. 225-242. 
MOORE, J. K.; BRAYMER, H. D.; LARSON, A. D. Isolation of a Pseudomonas sp. which utilizes the phosphonate herbicide glyphosate. Appl. Environ. Microbiol., v. 46, p. 316-320, 1983.

MOORMAN, T. B.; DOWLER, C. C. Herbicide and rotation effects on soil and rhizosphere microorganisms and crop yields. Agric. Ecos. Environ., v. 35, p. 311-325, 1991.

PACOVSKY, R. S.; PAUL, E. A.; BETHLENFALVAY, G. J. Response of mycorrhizal and P fertilized soybeans to nodulation by Bradyrhizobium or ammonium nitrate. Crop Sci., v. 26, n. 1, p. 145-150, 1986.

PAULA, M. A. at al. Efetividade simbiótica relativa em soja de populações de fungos endomicorrízicos nativos e de isolados de Glomus macrocarpum e Gigaspora margarita. R. Bras. Ci. Solo, v. 12, n. 1, p. 25-31, 1988.

PAULA Jr, T. J.; ZAMBOLIM, L. Efeito de fungicidas e de herbicidas sobre a micorrização de Eucalyptus grandis por Glomus etunicatum. Fitopatol. Bras., v. 19, n. 2, p. 173177, 1994.

SOUZA, R. F. et al. Micorriza e fósforo no crescimento de Brachiaria brizantha e Stylosanthes guianensis em solo de baixa fertilidade. 2. Acúmulo de minerais. Past. Trop., v. 21, n. 3, p. 24-30, 1999.
SANTOS, J. B. et al. Comportamento de estirpes de Bradyrhizobium sp. sob efeito de componentes do glyphosate potássico. Rev. Ci. Agron., v. 34, n. 2, p. 201-206, 2003.

SANTOS, J. B. et al. Efeitos de diferentes formulações comerciais de glyphosate sobre estirpes de Bradyrhizobium. Planta Daninha, v. 22, n. 2, p. 293-300, 2004.

SANTOS, J. B. et. al. Tolerance of Brady rhizobium strains to Glyphosate formulations. Crop Protec., v. 24, p. 543-547, 2005.

SCHENCK, N. C. Methods and principles of mycorrhizal research. Saint Paul: The American Phytopatology Society Publication, 1982. 224 p.

TRAPPE, J. M.; MOLINA, R.; CASTELLANO, M. Reactions of mycorrhizal fungi and mycorrhiza formation to pesticides. Ann. Rev. Phytopathol., v. 22, p. 331-359, 1984.

ZIMMER, A.H.; EUCLIDES FILHO, K. As pastagens e a pecuária de corte brasileira. In: SIMPÓSIO INTERNACIONAL SOBRE PRODUÇÃO ANIMAL EM PASTEJO, 1997, Viçosa. Anais...Viçosa, MG: 1997. p. 349-379. 